\title{
Tetra-marching procedure for high order Level Contour Reconstruction Method
}

\author{
I. Yoon \& S. Shin \\ Department of Mechanical and System Design Engineering, \\ Hongik University, Korea
}

\begin{abstract}
Direct numerical simulation of the multiphase flow on a fixed Eulerian grid became increasingly popular due to its simplicity and robustness. Lately, there have been efforts to construct hybrids from existing well-known methods including VOF, Level Set, and Front Tracking with the intention of overcoming the inherent drawbacks of each method. The Level Contour Reconstruction Method is one of the hybrid type methods, which combines the Front Tracking and Level Set characteristics. By introducing a high order interpolation kernel during interface reconstruction, we could reconstruct the interface very accurately and smoothly compared to linear interpolation of a given distance function field. The high order reconstruction procedure can be undertaken, generally, with the same rectangular shaped Eulerian grid structure as the flow computation. With this rectangular shaped grid, there can be more than two lines in a single cell during the reconstruction. In this paper, we introduce the tetramarching reconstruction procedure which can eliminate ambiguity of drawing contour lines between multiple edge points in a single cell during high order reconstruction. We tested several benchmarking simulations of interface evolution and found that high order reconstruction with a tetra-marching procedure enables the fidelity of the reconstructed interface, which is continuous and smooth even with a highly distorted interface.
\end{abstract}

Keywords: interface reconstruction, front tracking, level set, tetra-marching, numerical simulation. 


\section{Introduction}

Multiphase flow is an ubiquitous process used for diverse engineering applications. Due to the small length and time scale associated with multiphase flow, a numerical technique can be an ideal tool for identifying underlying physics compared to experiment. Lately, methods using a fixed Eulerian grid with an additional advection scheme to track the interface motion, e.g. VOF [1], Level Set [2], and Front Tracking [3], has drawn great attentions. Despite the success of each method, it is still a formidable task to come up with an accurate, reliable, and yet simple method which can model complex interfacial dynamics, especially for three-dimensional simulation. The Level Contour Reconstruction Method (LCRM) [4-7] which combines the advantage of the Front Tracking and Level Set method has been devised to overcome some drawbacks pertaining to each method. Similar efforts can also be found in various hybrid type methods including CLSVOF(Level Set+VOF) from Sussman and Puckett [8], particle Level Set (Level Set+Lagrangian marker particle) from Enright et al. [9], and Aulisa et al. [10] who combines markers and the VOF method.

The LCRM is basically a Front Tracking type method which tracks implicitly connected individual interface elements (lines for 2D and faces for 3D). Implicit connectivity of the interface elements make it possible to eliminate the burden of bookkeeping of the logistic information of neighbouring elements which incurs a large memory and calculation cost. At the same time, it takes advantage of the Level Set method since the interface can also be represented by an Eulerian function field (e.g. distance function) which can be computed directly from a given interface location. Reconstruction of the interface at a certain level of the distance function enables us to model the merging and pinch off of interfaces naturally and automatically as in the Level Set method. During the original reconstruction procedure [4], the interface was relocated by linear interpolation from the given distance function field. We found that linear interpolation generates a continuous but not smooth interface after reconstruction. High order LCRM [6] has been introduced to increase the accuracy and smoothness of the reconstructed interface since a small disturbance originated from linear reconstruction can cause instability of the solution requiring frequent interface reconstruction, especially with a low resolution.

In general, the reconstruction procedure can be undertaken within the same rectangular shaped Eulerian grid structure as flow computation. In the case of highly deformable interface evolution, the phase front can elongate to form a thin filament and approach too close to each other. High order reconstruction makes it possible to relocate this subtle feature accurately but can also generate the ambiguity of drawing contour lines in a rectangular cell since there can be more than two lines with an attenuated interface. Without a complex algorithm, there can be small holes along, with a narrow gap of the elongated interface. This effect is usually negligible since reconstruction will be performed regularly but might cause solution instability where surface tension plays a dominant role. In this paper, we introduce the tetra-marching procedure which 
can eliminate ambiguity to draw lines between multiple edge points in a single cell during high order reconstruction.

\section{Numerical formulation}

\subsection{Governing equations}

The single field form of the governing equations for multi fluid motion including phase change can be expressed as:

$$
\begin{gathered}
\nabla \cdot \mathbf{u}=0 \\
\rho\left(\frac{\partial \mathbf{u}}{\partial t}+\mathbf{u} \cdot \nabla \mathbf{u}\right)=-\nabla p+\rho \mathbf{g}+\nabla \cdot \mu\left(\nabla \mathbf{u}+\nabla \mathbf{u}^{\mathrm{T}}\right)+\mathbf{F} \\
\frac{\partial T}{\partial t}+\mathbf{u} \cdot \nabla T=\alpha \nabla^{2} T\left(\begin{array}{c}
\alpha=\alpha_{L} \text { in liquid phase } \\
\alpha=\alpha_{G} \text { in gas phase }
\end{array}\right)
\end{gathered}
$$

here, $\mathbf{u}$ is the velocity, $p$ is the pressure, $\mathbf{g}$ is the gravitational acceleration, and $\mathbf{F}$ is the local surface tension force at the interface which can be described by the hybrid formulation $[5,7]$ as:

$$
\mathbf{F}=\sigma \kappa_{H} \nabla I
$$

where $\sigma$ is the surface tension coefficient (assumed constant here), I is the indicator function, a Heaviside function which varies from zero to one near the interface. The indicator function also has been utilized to describe material property at each phase. $\kappa_{\mathrm{H}}$ is the curvature field calculated on the Eulerian grid given by:

$$
\kappa_{H}=\frac{\mathbf{F}_{L} \cdot \mathbf{G}}{\sigma \mathbf{G} \cdot \mathbf{G}}
$$

where

$$
\begin{gathered}
\mathbf{F}_{L}=\int_{\Gamma(t)} \sigma \kappa_{f} \mathbf{n}_{f} \delta_{f}\left(\mathbf{x}-\mathbf{x}_{f}\right) \mathrm{d} s \\
\mathbf{G}=\int \mathbf{n}_{f} \delta_{f}\left(\mathbf{x}-\mathbf{x}_{f}\right) d s
\end{gathered}
$$

here, $\mathbf{n}_{f}$ is the unit normal from the interface, $\mathbf{x}_{f}$ is a location of the interface, and $\delta_{f}\left(\mathbf{x}-\mathbf{x}_{f}\right)$ is a dirac delta distribution that is non-zero only at the interface. $d s$ is the length or area of the element, and $\kappa_{f}$ is twice the mean interface curvature computed in a Lagrangian fashion.

The indicator function can be obtained using a distance function which has been computed directly from a given interface front [7]. This additional hybridization with the Level Set technique enables us to compute an accurate distance function as well as to locate the minimum distant point on the interface from grid node of interest. This information then allows us to calculate a 
compact curvature field based on the curvature at a minimum distance point on the interface. This compact curvature field can further reduce the parasitic current to a significantly lower level [7]. The more detailed procedure for calculating the compact curvature field can be found in [4-7].

The interface is advected in a Lagrangian fashion by integrating

$$
\frac{\mathrm{d} \mathbf{x}_{f}}{\mathrm{~d} t}=\mathbf{V}
$$

where $\mathbf{V}$ is the interface velocity vector interpolated at $\mathbf{x}_{f}$. The energy equation (eqn (3)) has been solved using the sharp interface technique [11] and a detailed solution procedure and discretization of the governing equations can be found in [4-7].
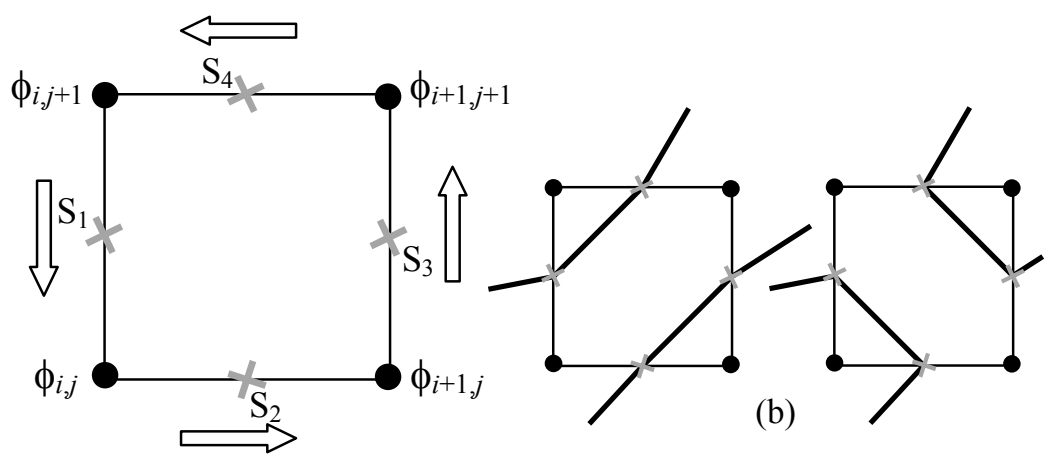

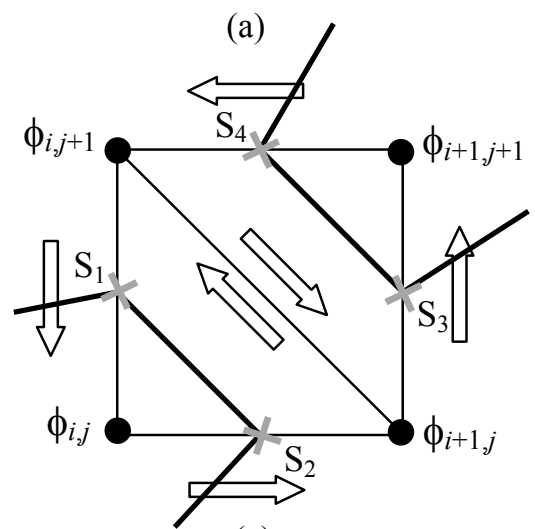

(c)

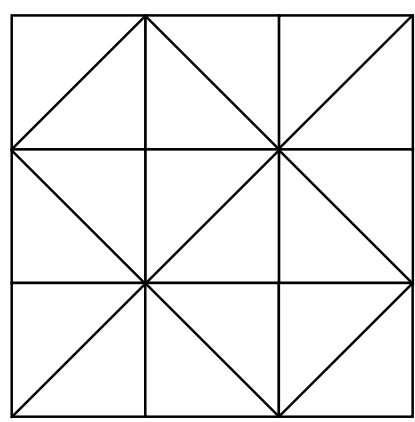

(d)

Figure 1: Two-dimensional interface generation procedure for the LCRM (a) original LCRM using a rectangular cell (b) ambiguity in the interface reconstruction with a rectangular cell (c) tetra-marching reconstruction with a triangular cell (d) alternative orientation of triangular cells for interface connectivity. 


\subsection{High order reconstruction method}

The original LCRM [4] used linear interpolation kernel to create contour lines or faces from given indicator or distance function field. Here we will briefly explain the basic procedure of the original LCRM in 2D. As you can see from fig. 1, we try to search the specific contour level of the function $\phi$, e.g. zero value in the case of distance function, along with the grid cell edges. If we find two points on the edges of the grid cell $\left(\phi_{i, j} \phi_{i, j+1}<0\right.$ and $\left.\phi_{i, j} \phi_{i+1, j}<0\right)$, we draw a contour line between those two points $\left(\mathrm{S}_{1}\right.$ and $\left.\mathrm{S}_{2}\right)$. After sweeping all grid cells considered, the reconstructed interfaces will share the same end points thus we can achieve implicit connectivity of the interface.

We found that linear interpolation generates a small disturbance since it cannot guarantee smoothness of the interface but only connectivity. Moreover, mass can redistribute from the high curvature region to the lower curvature region by enforcing global mass conservation during interface reconstruction. These slight discrepancies in the interface points can induce undesirable results where the exact location of the interface is extremely important. Thus high order reconstruction [6] was introduced and we will briefly summarize the main idea.

The distance function $\phi(i, j)$ (fig. 1(a)) where the specific value, i.e. zero, represents the interface position can be modified by adding a trial function, $\psi(i, j)$, as follows:

$$
\phi^{H}(\mathbf{x})=\sum_{g}[\phi(i, j)+\psi(i, j)] S\left(\mathbf{x}-\mathbf{x}_{g}\right)
$$

here, $\mathbf{x}$ is the evaluation point, $\mathbf{x}_{\mathrm{g}}$ is the grid cell centre, $S\left(\mathbf{x}-\mathbf{x}_{\mathrm{g}}\right)$ is the interpolation kernel composed of B-splines for smoothness of function values, suggested by Torres and Brackbill [12]. The summation is performed across a small multiple of the mesh, usually four grid cells wide, in each $x, y$ direction.

The trial function has been added since the zero contour level of the original distance function itself cannot describe exactly the original interface location. We have used the same B-spline type interpolation kernel for trial function:

$$
\psi(i, j)=\sum_{N_{p}} \delta I_{p} S\left(\mathbf{x}_{g}-\mathbf{x}_{p}\right)
$$

here, $\mathbf{x}_{\mathrm{p}}$ is the location of the original interface points before reconstruction, $\delta I_{p}$ is the increment needed at the original interface points, and the integral has been performed over all interface elements. This trial function makes the zero contour level of eqn (9) lies exactly on top of the original interface location. The method is very simple and has a comparable accuracy to other methods using complicated smoothing procedures. The basic idea of high order LCRM is similar to the Point Set method of Torres and Brackbill [12] except that their indicator function has been computed directly from the interface elements. The detailed procedure for high order reconstruction can be found in [6]. 


\subsection{Tetra-marching procedure}

The reconstruction procedure can be undertaken, in general, with the same rectangular shaped Eulerian grid structure as flow computation. With this rectangular shaped grid, there can be more than four points $\left(S_{1}, S_{2}, S_{3}\right.$, and $\left.S_{4}\right)$ at four edges of the reconstruction cell as described in fig. 1(a) when the interface stretches severely to form a thin filament $\left(\phi_{i, j+1}>0, \phi_{i+1, j}>0, \phi_{i, j}<0, \phi_{i+1, j+1}<0\right)$. In this case, there can be ambiguity of drawing contour lines in a single cell during the reconstruction procedure as in fig. 1(b). Special formulation of the interface reconstruction is required for the rectangular shaped reconstruction domain. For two-dimensional simulation, this can be done with a relatively simple procedure but it will be an overwhelming task for three-dimensional simulation.

Drawing a line or surface from given data is an iso-surface extraction process which has been a major concern for volume visualization of the scattered data set from a medical image such as a CT and MRI scan. Marching cubes technique [13] is the most popular procedure for extracting surface from cubical grid data. 256 cube configurations are possible but it can be reduced to 15 unique cases using symmetry and reflective condition. The look-up table has been generated with vertex data then reconstruction has been performed to each cube in a marching direction.

Lately, Carneiro et al [14] proposed the tetra-cubes reconstruction method which does not use a look-up table to extract iso-surface data from a medical image thus eliminating the choice from 15 possible interfacial geometries in the case of rectangular shaped geometry. Similarly, interface reconstruction does not have to coincide with the rectangular grid cell as primary variables of velocity and pressure for the LCRM. We will start with 2D reconstruction for simplicity. The ambiguity of drawing lines from multiple edge points can be avoided by using a triangular reconstruction grid as in fig. 1(c). There can be only one line from each triangular cell of interest for $2 \mathrm{D}$ simulation. We also need to make the reconstructed interface share the same end points between neighbouring cells. To make the reconstructed interface continuous, a triangular grid has been oriented in an alternative fashion as shown in fig. 1(d).

Three-dimensional reconstruction is basically the same as a two-dimensional one. As can be seen from fig. 2 (a), we first reconstructed edge lines from 6 faces wrapping the cubic cell. These lines will compose the line edges of the reconstructed 2D surface element. As pointed out earlier [13], there can be 15 possibilities of interface location with a given distance function value at 8 vertices. This problem can be also avoided by using a tetrahedral mesh [14] instead of a cubic one. In the case of the tetrahedral cell, there can be two possible interface geometries after reconstruction as in fig. 2(b). The first one is the triangular element which does not need any modification. The second is the rectangular element which has been modified to triangles by subdividing in the direction of the longest distance between edge points. Even if we use a tetrahedral cell for interface reconstruction, we still need to orient the tetrahedral cell carefully to guarantee connectivity of the reconstructed interface elements. We try to use a given Cartesian cubical cell for fluid computation as our basic 
reconstruction cells and subdivide this to five tetrahedral cells as in fig. 3(a). The five sub-cells have been reoriented to match the reconstructed edges at cube faces of the neighbouring cell as in the two-dimensional case (fig. 3(b)). By using zigzag orientation of the tetrahedral cells [14], we can construct continuous surface elements after reconstruction.

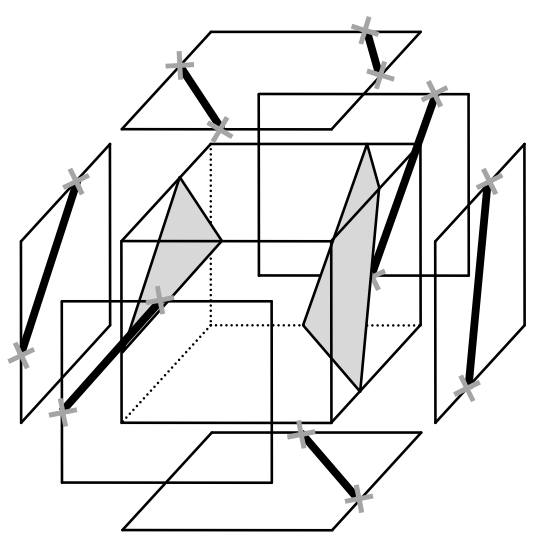

(a)

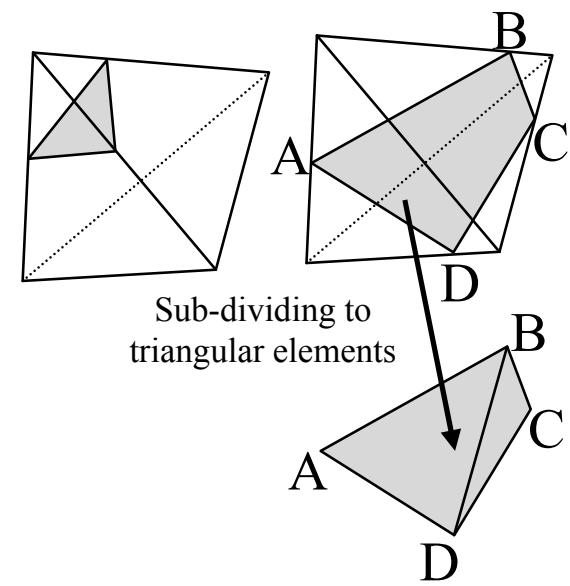

(b)

Figure 2: Three-dimensional interface generation procedure for the LCRM (a) original LCRM using a Cartesian rectangular cell (b) tetramarching reconstruction using a tetrahedral cell.

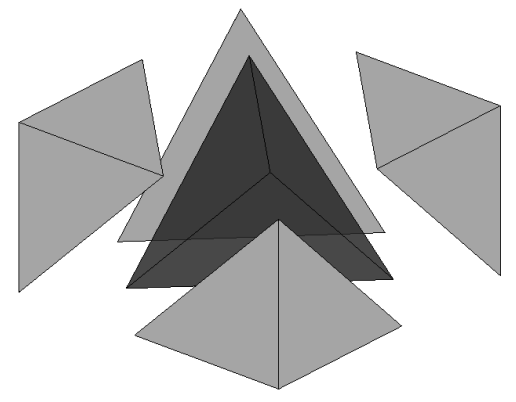

(a)

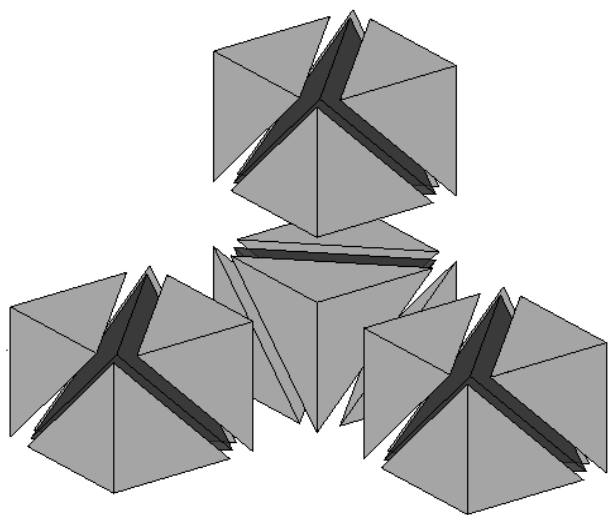

(b)

Figure 3: Three-dimensional interface generation procedure for the LCRM (a) tetrahedral cell generation from a rectangular cell (b) zigzag orientation of a tetrahedral cell for interface connectivity. 


\section{Results and discussion}

\subsection{Geometry tracking test}

We first tested the "vortex in a box" problem introduced by Bell et al [15]. The test can demonstrate the method's ability to accurately resolve thin filaments on the scale of a grid cell. A circle of radius 0.15 is placed at $(0.5,0.75)$ using a $64 \times 64$ grid resolution in unit domain. The velocity field is defined by:

$$
\mathbf{v}(\mathbf{x})=2\left[\begin{array}{c}
-\sin ^{2}(\pi x) \sin (\pi y) \cos (\pi y) \\
\sin ^{2}(\pi y) \sin (\pi x) \cos (\pi x)
\end{array}\right]
$$

The resulting velocity field stretches out the circle into a very long, thin spiral filament which progressively wraps itself toward the centre of the box. In this case the interface elements become highly elongated by the flow and a reconstruction procedure becomes essential for an accurate simulation. Fig. 4 shows the interface at $t=3$. Reconstruction has been performed at every $100^{\text {th }}$ time step and a total number of 30 reconstructions have been performed for the simulation. As can be seen from fig. 4(a), the fine structure of the interface has been successfully captured but the reconstructed interface does not have complete connectivity near the thin filamentary region using a rectangular shaped reconstruction cell with high order reconstruction. We have not provided any logical algorithm when ambiguity of drawing interface arises. Fig. 4(b) shows tetra-marching reconstruction of the interface at an identical time as in Fig. 4 (a). The interface has been well connected without any additional algorithm.

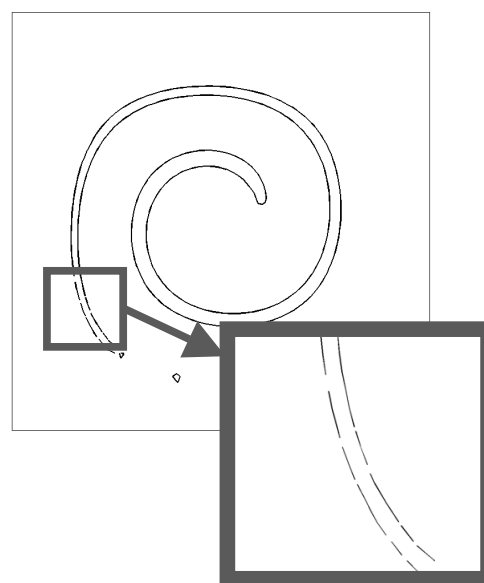

(a)

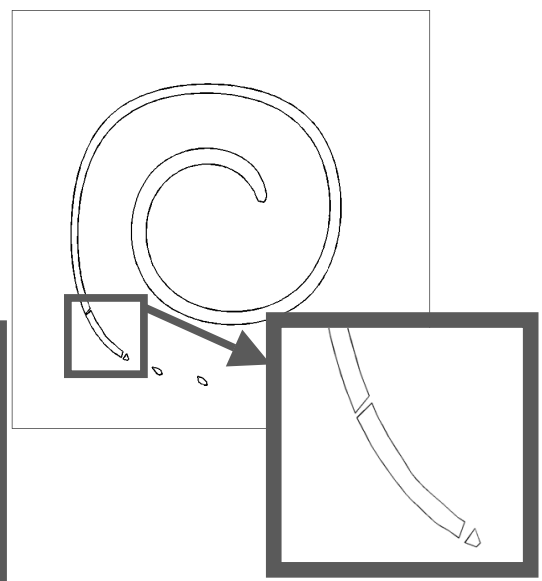

(b)

Figure 4: Comparison of reconstruction accuracy (a) original LCRM using rectangular cell (b) tetra-marching reconstruction using triangular cell. 


\subsection{Nucleate boiling with convective flow}

We have simulated nucleate boiling with fluid convection to validate reconstruction accuracy during severe deformation of the interface in 3D. The governing equations (1) to (3) can be non-dimensionalized by appropriate scales of length $l_{0}=\left(\sigma / g\left(\rho_{L^{-}} \rho_{G}\right)\right)^{1 / 2}$, velocity $U_{0}=\left(g l_{0}\right)^{1 / 2}$, temperature $\rho_{\mathrm{G}} L_{o} / \rho_{\mathrm{L}} \mathrm{c}_{\mathrm{L}}$ (measured from $T_{\text {sat }}$ ), and pressure $\rho_{\mathrm{L}} U_{\mathrm{o}}^{2}$ (measured from the ambient system pressure $P_{\infty}$ ). The problem can then be characterized by the Reynolds, $R e$, Peclet, $P e$, Jakob, $J a$ and Weber, We, numbers as well as the property ratios which can be defined as follows:

$$
\begin{gathered}
R e=\frac{\rho_{L} U_{o} l_{o}}{\mu_{L}}, W e=\frac{\rho_{L} U_{o}^{2} l_{o}}{\sigma}, P e=\frac{k_{L}}{\rho_{L} c_{L} U_{o} l_{o}}, J a=\frac{T_{s a t} c_{L}}{L_{o}} \\
\rho^{*}=\frac{\rho_{G}}{\rho_{L}}, k^{*}=\frac{k_{G}}{k_{L}}, \mu^{*}=\frac{\mu_{G}}{\mu_{L}}, c^{*}=\frac{c_{G}}{c_{L}}
\end{gathered}
$$

here $\rho$ is density, $\mu$ is viscosity, $k$ is thermal conductivity, $c$ is specific heat, $T$ is temperature and $L_{o}$ is the latent heat of vaporization. A more detailed description of numerical formulation can be found in $[4,16]$

During nucleate boiling, individual bubbles will directly contact the heated wall so proper modelling of the contact line motion becomes very important. The detailed physics of the contact region regarding contact line motion is still poorly understood thus we applied a simple Navier-slip model [11]. The interface can move freely between the advancing and receding angle and the contact angle has been fixed to a constant angle of $120^{\circ}$ if the contact angle is greater than the prescribed advancing angle of $120^{\circ}$ and, vice versa, to a receding angle of $60^{\circ}$. We also used a simplified form of the microlayer evaporation model proposed by Son [17] which uses algebraic formulation based on geometric constraint instead of solving a nonlinear differential equation.

Initially, four hemispherical cap shaped bubbles in different sizes of 1.0, 1.05, 1.1 , and 1.15 are placed at each centre of the four quadrants of the bottom wall where a no-slip velocity boundary condition has been used (small window of fig. 5). A periodic boundary condition has been applied to both the $x$ and $y$ direction and moving wall boundary condition with $3 \mathrm{~m} / \mathrm{s}$ in the $x$ direction is used for the top wall. To allow vaporization, fluid is also allowed to exit at the top boundary where the pressure is specified to be zero. The temperature field is initially zero everywhere except the rigid bottom wall where a constant non-dimensional temperature of 108 applied. Temperature at the top wall boundary has been specified as -532 . We have chosen to use the properties of water at atmospheric condition thus our simulation parameters will be $\rho^{*}=0.0006237, \mu^{*}=0.04353$, $k^{*}=0.03694, c^{*}=0.4847, R e=1334.2, W e=1.0, P e=0.0004268$, and $J a=0.6972$, The box size of $4.2 \times 4.2 \times 6.3$ with a $40 \times 40 \times 60$ grid resolution has been used for the simulation. 


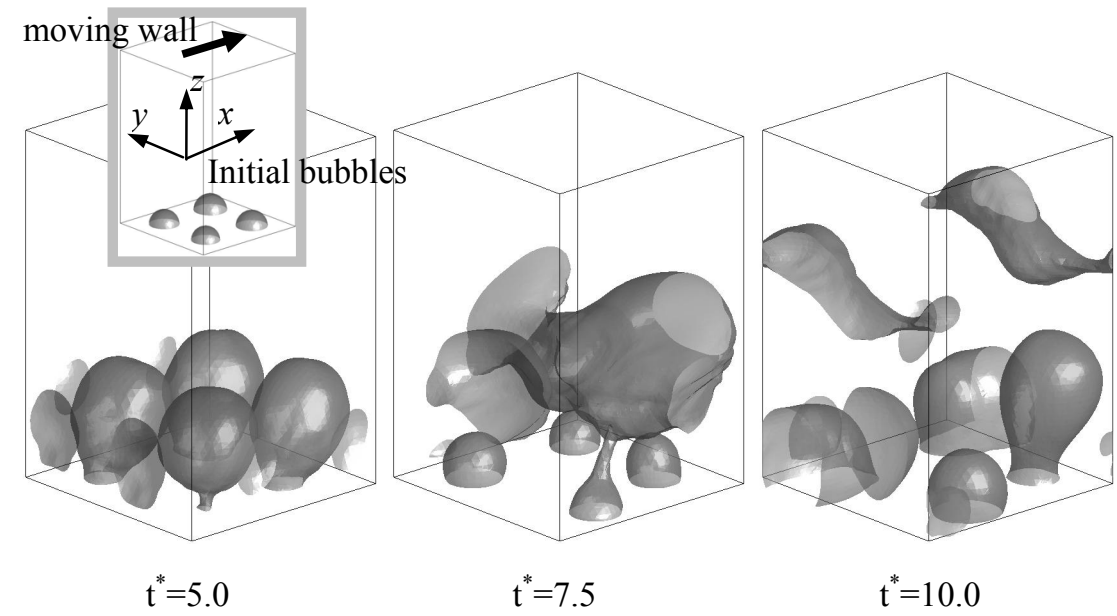

Figure 5: Nucleate boiling with multiple bubbles under fluid convection.

As can be seen from fig. 5, multiple bubbles are growing independently at the start and merging to form a larger bubble. A merged bubble is finally pinched off from the bottom surface then experiencing the condensation from the top boundary temperature condition. The interface undergoes severe transition of the topological evolution. At $t^{*}=7.5$, there exists a thin filamentary vapour column right before pinch off and the tetra-marching procedure can generate a closed interface after reconstruction. There is also a complicated merging process of the interfaces at the middle section of the domain. We found that the tetra-marching reconstruction procedure can generate perfectly interconnected elements as well as a naturally and automatically merged interface after reconstruction. More quantitative research of nucleate boiling including surface heat transfer is necessary and currently underway.

\section{Conclusion}

The LCRM is one of the hybrid type interface methods for multiphase flows which combines some essential features of the Front Tracking and Level Set techniques. While retaining an explicitly tracked interface using interfacial elements, the distance function calculated from a given interface location plays a crucial role in periodic reconstruction of the interface elements.

By adopting high order reconstruction, we can maintain an excellent mass conservation and interface fidelity. However, there can be ambiguity during interface reconstruction in the case of a rectangular shaped reconstruction cell. Despite the fact that this local disconnection of the interface will not cause complete breakdown of the simulation, it generates a relatively large error where the interface evolves into a thin filamentary shape. The tetra-marching procedure can create a perfectly interconnected interface near the very tail of a filamentary 
shaped interface. The procedure is simple and easily applicable for both $2 \mathrm{D}$ and 3D simulations without any complex algorithm. It can handle complicated interface evolution in a straightforward manner. Several tests including interface evolution in a vortex field and three-dimensional nucleate boiling problems with convective flow with a large surface deformation have been performed and the tetra-marching reconstruction procedure showed an accurate reconstruction capability compared to the original method.

\section{References}

[1] Scardovelli, R. \& Zaleski, S., Direct numerical simulation of free-surface and interfacial flow, Ann. Rev. Fluid Mech., 31 , pp 567-603, 1999

[2] Osher, S. \& Fedkiw, R.P., Level set methods: An overview and some recent results, J. Comput. Phys., 169, pp 463-502, 2001.

[3] Tryggvason, G., Bunner, B., Esmaeeli, A., Juric, D., Al-Rawahi, N., Tauber, W., Han, J., Nas, S. \& Jan, Y.J., A front tracking method for the computations of multiphase flow, J. Comput. Phys. 2001; 169: 708-759.

[4] Shin, S. \& Juric, D., Modeling three-dimensional multiphase flow using a level contour reconstruction method for front tracking without connectivity, J. Comput. Phys., 180, pp 427-470, 2002.

[5] Shin, S., Abdel-Khalik, S.I., Daru, V. \& Juric, D., Accurate representation of surface tension using the level contour reconstruction method, $J$. Comput. Phys., 203, pp 493-516, 2005.

[6] Shin, S. \& Juric, D., High order level contour reconstruction method, Journal of Mechanical Science and Technology, 21, pp 311-326, 2007.

[7] Shin, S., \& Juric, D., A hybrid interface method for three-dimensional multiphase flows based on front tracking and level set technique, Int. J. Numerical Methods in Fluids, 60(7), pp 753-778, 2009.

[8] Sussman, M. \& Puckett, E.G., A coupled level set and volume-of-fluid method for computing 3D and axisymmetric incompressible two-phase flows, J. Comput. Phys., 162, pp 301-337, 2000.

[9] Enright, D., Fedkiw, R., Ferziger, J. \& Mitchell, I., A hybrid particle level set method for improved interface capturing, J. Comput. Phys., 183, pp 83$116,2002$.

[10] Aulisa, E., Manservisi, S. \& Scardovelli, R., A mixed markers and volumeof-fluid method for the reconstruction and advection of interfaces in twophase and free-boundary flows, J. Comput. Phys., 188, pp 611-639, 2003.

[11] Liu, H., Krishnan, S., Marella, S. \& Udaykumar, H. S., Sharp interface Cartesian grid method II: a technique for simulating droplet interactions with surfaces of arbitrary shape, J. Comput. Phys., 210, pp 32-54, 2005.

[12] Torres, D.J. \& Brackbill, J.U., The point-set method: front-tracking without connectivity, J. Comput. Phys., 165, pp 620-644, 2000.

[13] Timothy, S.N. \& Hong, Y., A survey of the marching cubes algorithm, Computers and Graphics, 30, pp 854-879, 2006 
[14] Carneiro, B.P., Silva, C.T. \& Kaufman, A.E., Tetra-Cubes: an algorithm to generate $3 \mathrm{D}$ isosurfaces based upon tetrahedral, Anais do IX SIBGRAPI, pp 205-210, 1996.

[15] Bell, J.B., Colella, P. \& Glaz, H.M., A second-order projection method for two-phase flow consisting of separate compressible and incompressible regions, J. Comput. Phys., 85, pp 257-283, 1989.

[16] Juric, D. \& Tryggvason, G., Computations of boiling flows, Int. J. Multiphase Flow, 24, pp 387-410, 1998.

[17] Son, G., Numerical simulation of bubble motion during nucleate boiling, Transaction of the KSME B., 25(3), pp 389-396, 2001. 